\title{
Conceitos Fundamentais em Espectroscopia
}

\author{
Adalberto B. M. S. Bassi * \\ bassi@iqm.unicamp.br \\ Universidade Estadual de Campinas, Instituto de Química
}

\section{Informações do Artigo Resumo}

Histórico do Artigo Os efeitos resultantes da interação de radiações eletromagnéticas com a Criado em Setembro de 2001 matéria proporcionam evidências do comportamento microscópico. Estas observações levam-nos a sugerir modelos que permitam compreender ou prever as propriedades do material estudado. Usualmente este modelos estão associados com princípios e conceitos associados a mecânica quântica. Neste tópico serão apresentados os conceitos elementares necessários para a compreensão dos efeitos espectroscópicos associados com o fenômeno de absorção e emissão de luz.

\author{
Palavras-Chaves \\ Espectroscopia \\ Aproximação de Born-Oppenheimer \\ Radiação \\ Densidade de energia \\ Coeficientes de Einstein \\ Lei de Beer-Lambert \\ Regras de seleção \\ Potencial de Morse \\ Princípio de Franck-Condon
}

\section{A Aproximação de Born-Oppenheimer}

O movimento de translação, por definição, ocorre homogeneamente na molécula como um todo, ou seja, não modifica as posições relativas das partículas que a constituem. Se os núcleos formassem uma estrutura rígida, tal estrutura poderia girar, ao que se chamaria rotação molecular. Entretanto, as distâncias internucleares e os ângulos entre as ligações vibram, enquanto que a estrutura nuclear, como um todo, gira. Ao movimento resultante desta superposição e interação de movimentos denomina-se roto-vibração molecular. As velocidades típicas associadas à roto-vibração são inferiores à do som $\left(340 \mathrm{~m} \mathrm{~s}^{-1}\right)$. Os elétrons de valência apresentam velocidades $\mathrm{da}$ ordem de $10^{6} \mathrm{~m} \mathrm{~s}^{-1}$ e os elétrons internos velocidades maiores, podendo até se aproximarem da velocidade da $\operatorname{luz}\left(\mathrm{c}=3 \times 10^{8} \mathrm{~m} \mathrm{~s}^{-1}\right)$.

O movimento relativo elétrons-núcleos reflete, evidentemente, o fato de ambos se moverem em relação a algum referencial fixo. Entretanto, como as velocidades eletrônicas são pelo menos mil vezes maiores do que as nucleares, é uma aproximação razoável desprezar a roto-vibração ao se considerar o movimento dos elétrons em relação aos núcleos (ou dos núcleos em relação aos elétrons).

\footnotetext{
Agradecimentos

Por apoio e sugestões, aos Profs. Drs. Edvaldo Sabadini, Francisco B. T. Pessine, Teresa D. Z. Atvars e Watson Loh e Claudia Martelli.
} 
Em outras palavras, é uma aproximação razoável considerar que, a cada momento ao longo do movimento roto-vibracional, a distribuição eletrônica espacial instantaneamente se ajusta às correspondentes posições nucleares, de modo a manter sempre minima a energia molecular. Esta é a aproximação de Born-Oppenheimer, que permite resolver a equação de Schrödinger, para o movimento eletrônico em relação aos núcleos de uma molécula, considerando estes últimos fixos. Por outro lado, ela permite que a equação de Schrödinger para o movimento nuclear seja resolvida considerando-se a influência eletrônica como um potencial dependente das posições nucleares. Esta aproximação produz uma imensa simplificação matemática no estudo mecânico-quântico das moléculas, ao preço de introduzir nos resultados um erro geralmente desprezível quando comparado com outros, criados por outras aproximações frequentemente usadas.

\section{A Equação Fundamental}

Considerando a aproximação de Born-Oppenheimer e excluíndo a energia translacional, o número de onda correspondente à energia total de uma molécula diatômica pode ser escrito

$\widetilde{v}_{t o t}=\widetilde{v}_{e l}+\widetilde{v}_{e}\left(\mathrm{v}+\frac{1}{2}\right)-\widetilde{x}_{e} \widetilde{v}_{e}\left(\mathrm{v}+\frac{1}{2}\right)^{2}+\widetilde{B}_{\mathrm{v}} J(J+1)-\widetilde{D} J^{2}(J+1)^{2}$

, em que:

- $\widetilde{\mathrm{v}}_{t o t}=E_{t o t} / h c$, sendo J a unidade de $E_{t o t}$ e J.cm a do produto $h c$ (a constante de Planck é $\mathrm{h}=6,626 \times 10^{-34}$ $\mathrm{J}$ s e a velocidade da luz é c $=3,000 \times 10^{10} \mathrm{~cm} \cdot \mathrm{s}^{-1}$, logo a $\widetilde{v}_{t o t}$ de é $\mathrm{cm}^{-1}$. Evidentemente, portanto, a unidade de todos os termos da equação anterior é $\mathrm{cm}^{-1}$. O módulo

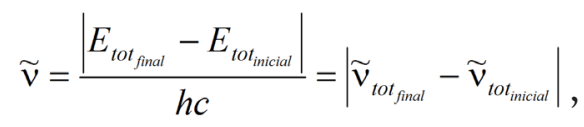

numa transição radioativa (radioativa significa que absorve ou emite radiação eletromagnética), é o número de onda absorvido (quando $\widetilde{v}_{t o t_{\text {frat }}}>\widetilde{v}_{\text {tot timical }}$ ) ou o número de onda emitido (quando $\widetilde{v}_{\text {tot trinal }}<\widetilde{v}_{\text {tot } \text { timcial }}$ ). Note-se que, neste resumo, sempre que o símbolo $\widetilde{v}$ apresentar um índice alfabético ele se referirá a um número de onda relacionado a alguma energia molecular especificada pelo índice (tot para total, el para eletrônica e $e$ para vibracional). Por outro lado, sempre que $\widetilde{V}$ não apresentar índice, ou apresentar índice não alfabético, ele se referirá ao número de onda de alguma radiação absorvida ou emitida.

1. $\widetilde{v}_{e l}=E_{e l} /$ hc é o número de onda correspondente a $E_{e l}$, sendo esta última a energia do mínimo da curva considerada para descrever a energia potencial eletrônica em função da distância internuclear (veja o item 1.1), assim como $\widetilde{\mathrm{v}}_{t o t}$ é o número de onda correspondente a $E_{t o t}$. Notese que, por causa do princípio de incerteza de Heisenberg, a energia da roto-vibração molecular não pode ser nula, logo a energia total mínima é obrigatoriamente superior a $E_{e p}$ ou seja,

$$
\widetilde{\mathrm{v}}_{\text {tot mínimo }}>\widetilde{\mathrm{v}}_{e l} \text {. }
$$

É conveniente definir o inteiro não negativo adimensional $\mathrm{n}=0,1,2, \ldots$, que numera as curvas de energia potencial eletrônica na ordem crescente dos valores de $\widetilde{v}_{e l}$.

- $\quad \widetilde{\mathrm{v}}_{e}=v_{e} / c$ é o número de onda correspondente à freqüência $v_{e}$ de vibração dos núcleos da molécula diatômica. $\mathrm{O}$ valor de $\mathrm{V}_{e}$ depende de qual é a curva de energia potencial eletrônica considerada para a molécula diatômica (ou seja, depende do valor de $n$ ), sendo único para cada uma destas curvas.

- $\quad \mathrm{v}=0,1,2, \ldots$ é um inteiro não negativo adimensional chamado número quântico vibracional que, junto com $n$, indica qual é o nível vibrônico em que o sistema se encontra. A energia de uma vibração depende de sua amplitude e do valor assumido por $\widetilde{v}_{e}$ mas, como já afirmado, o segundo não se altera quando $n$ não muda. Por isto, fixando-se uma curva de energia potencial eletrônica, a energia vibracional variará apenas com a amplitude, ambas quantizadas por $\mathrm{v}$ (note-se que a energia e a amplitude vibracionais mínimas são, obrigatoriamente, superiores a zero). Quando somente uma curva de energia potencial eletrônica estiver sendo considerada (geralmente aquela com menor valor de $\widetilde{v}_{e l}$, numerada $n=0$ e denominada fundamental), o nível vibrônico da molécula será denominado apenas vibracional.

- $\widetilde{x}_{e}$ é um adimensional não negativo denominado constante de anarmonicidade, responsável pela principal correção ao modelo do oscilador harmônico. Note-se que o termo $\widetilde{v}_{e}(v+1 / 2)$ fornece a energia correspondente a este modelo dividida por $b c$, enquanto que a subtração do termo $\widetilde{x}_{e} \widetilde{v}_{e}(v+1 / 2)^{2}$ corrige tal valor (correções de menor valor existem, mas foram desprezadas na equação anterior). Note-se também que, assim como $\widetilde{v}_{e}$, o valor de $\widetilde{x}_{e}$ depende de qual é o valor de $n$, sendo único para cada um destes 
valores. Se o zero de energia vibracional for $E_{e l}$, a energia vibracional da molécula será, portanto, dada por

$$
G(\mathrm{v})=h c\left[\widetilde{v}_{e}\left(\mathrm{v}+\frac{1}{2}\right)-\widetilde{x}_{e} \widetilde{v}_{e}\left(\mathrm{v}+\frac{1}{2}\right)^{2}\right] .
$$

- $B_{\mathrm{v}}=h / 8 \pi^{2} c \mu R_{e_{\mathrm{v}}}^{2}$ é a constante rotacional $\mathrm{em}^{-1}$, convencionando-se que $\mu=m_{1} m_{2} /\left(m_{1}+m_{2}\right)$ é a massa reduzida da molécula diatômica cujas massas atômicas são $m_{1}$ e $m_{2}$ e $R_{e_{v}}$ é a distância internuclear de equilíbrio para o nível vibrônico $(n, v)$ considerado, logo $\mu R_{e_{v}}^{2}$ é o momento de inércia da molécula. $O$ valor de $\widetilde{B}_{\mathrm{v}}$ depende, através de $R_{e_{v}}$, de qual é o nível vibrônico da molécula diatômica, sendo único em cada um destes níveis. Esta dependência é geralmente expressa por

$$
\widetilde{B}_{\mathrm{v}}=\widetilde{B}_{e}-\widetilde{\alpha}_{e}\left(\mathrm{v}+\frac{1}{2}\right)
$$

, onde

$\widetilde{B}_{e}$ e $\widetilde{\alpha}_{e}$ são grandezas não negativas que dependem apenas do valor de $\mathrm{n}$, sendo respectivamente únicas para cada um destes valores. Portanto, se a constante de interação vibração-rotação $\widetilde{\alpha}_{e}$ for nula, $\widetilde{B}_{\mathrm{v}}=\widetilde{B}_{e}$ e $R_{e_{\mathrm{v}}}$ $=R_{e}$, ou seja, $\widetilde{B}_{\mathrm{v}}$ e $R_{e_{\mathrm{v}}}$ dependerão apenas de $n$. Esta constante será nula quando cada ramo da curva de energia potencial eletrônica for a imagem especular do outro, como por exemplo acontece no caso da parábola que representa o potencial do oscilador harmônico (Figura 1), porque teremos então $R_{e_{\mathrm{v}}}=R_{e}$ , ou seja, a distância internuclear de equilíbrio será igual para todos os níveis vibrônicos de uma mesma curva. Para o oscilador anarmônico, entretanto, a forma dos dois ramos diferirá cada vez mais, a medida que $\mathrm{v}$ aumentar (Figura 2).

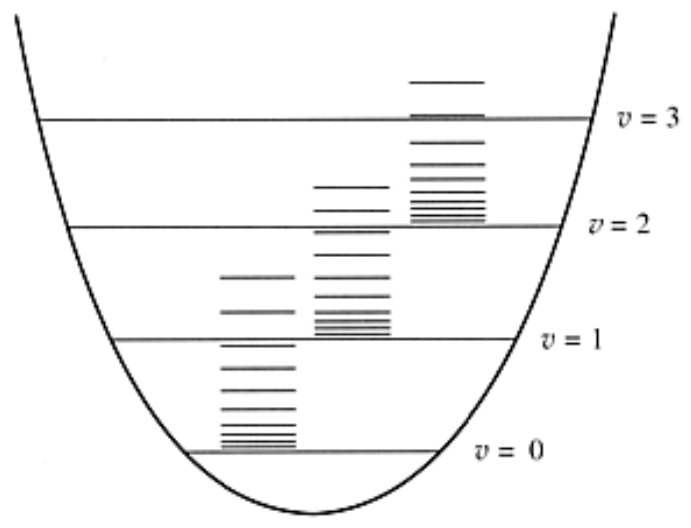

Figura 1 - Diagrama de energia mostrando os níveis rotacionais associados a cada nível vibracional para uma molécula diatômica em um determinado estado eletrônico.

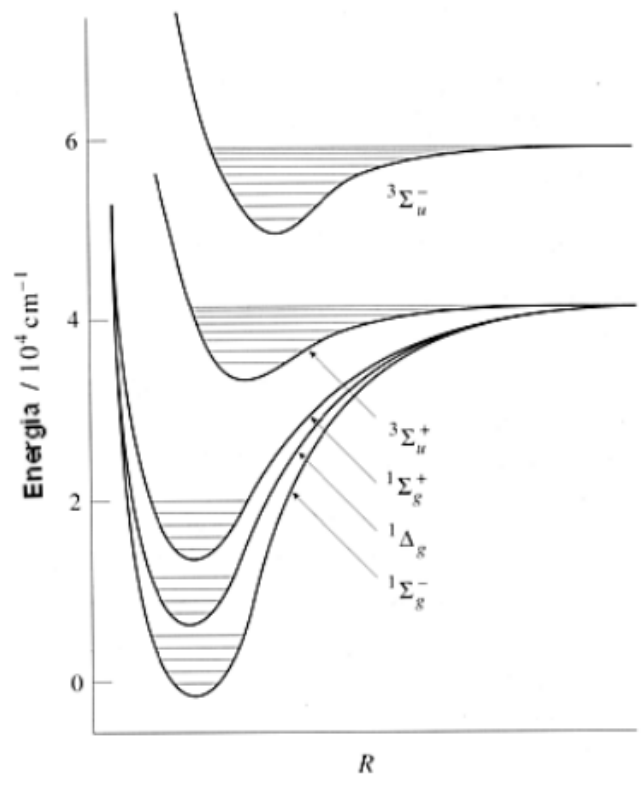

Figura 2 - Diagrama de energia da molécula de $\mathrm{O} 2$, mostrando os estados vibracionais associados aos vários estados eletrônicos.

- $\mathrm{J}=0,1,2, \ldots$ é um inteiro não negativo adimensional chamado número quântico rotacional que, junto com $\mathrm{n}$ e $\mathrm{v}$, indica qual é o nível roto-vibrônico em que o sistema se encontra. A energia de uma rotação depende da sua velocidade angular e do seu momento de inércia mas, como já afirmado, o segundo não se altera quando $\mathrm{n}$ e $\mathrm{v}$ não mudam. Por isto, fixando-se um nível vibronico de uma curva de energia potencial eletrônica, a energia rotacional variará apenas com a velocidade angular, ambas quantizadas por J (notese que a energia e a velocidade angular mínimas são iguais a zero). Quando somente uma curva de energia potencial eletrônica estiver sendo considerada (geralmente a fundamental), o nível roto-vibrônico da molécula será denominado apenas roto-vibracional.

- $\widetilde{D}$ é uma constante não negativa denominada distorção centrífuga, expressa $\mathrm{em} \mathrm{cm}^{-1}$, responsável pela principal correção ao modelo do rotor rígido. Sua existência provém do fato de que $R_{e_{v}}$ aumenta com $\mathrm{J}$, porque a ligação não é rígida, ou seja, $\widetilde{B}_{\mathrm{v}}$ e $R_{e_{\mathrm{v}}}$ dependem do nível roto-vibrônico e não apenas do nível vibrônico. Note-se que o termo $\widetilde{B}_{\mathrm{v}} J(J+1)$ fornece a energia do rotor rígido dividida por hc, enquanto que a subtração do termo $\widetilde{D} J^{2}(J+1)^{2}$ corrige tal valor, ou seja, permite que $\widetilde{B}_{\mathrm{v}}$ e $R_{e_{\mathrm{v}}}$ dependam apenas do nível vibrônico, como convencionalmente considerado (correções de menor ordem foram desprezadas na equação anterior). $\widetilde{D}$ depende somente do valor de $n$, sendo único para cada um destes valores. 
A Tabela 1 apresenta, para diversas moléculas diatômicas, valores dos parâmetros espectroscópicos antes descritos (o significado de $\widetilde{D}_{0}$ pode ser visto na Figura 3).

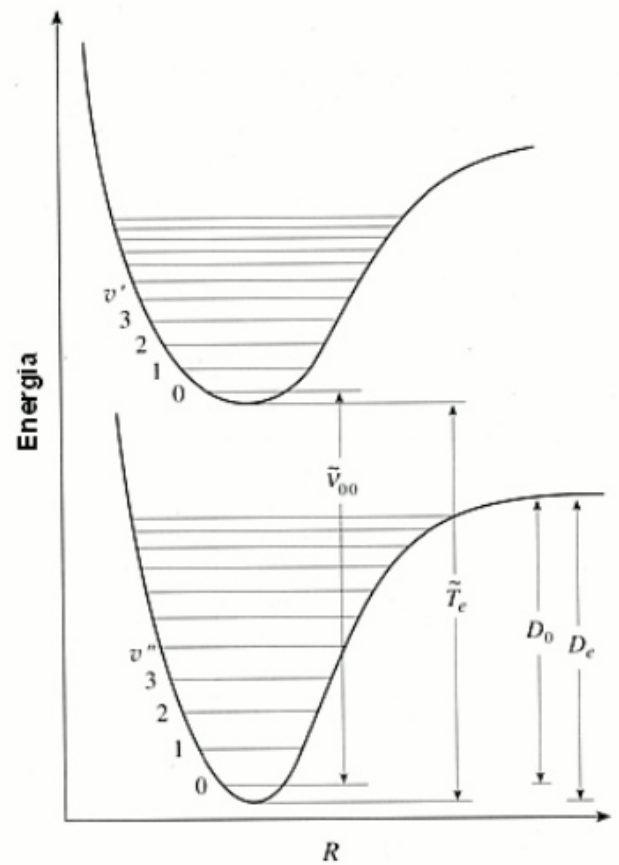

Figura 1 - Ilustração de dois estados eletrônicos de uma molécula diatômica. Observa-se a representação das quantidades $\widetilde{T}_{e}$ e $\widetilde{v}_{0,0}$

\section{A Equação Fundamental}

Intensidade de Radiação e Espectrofotômetro

Intensidade de radiação eletromagnética I é, por definição, a energia radiante que, por unidade de tempo, atravessa uma unidade de área ortogonal à direção de propagação da radiação. Quando moléculas de um material sofrem transição radioativa entre níveis de energia a elas permitidos, a intensidade da radiação monocromática com número de onda

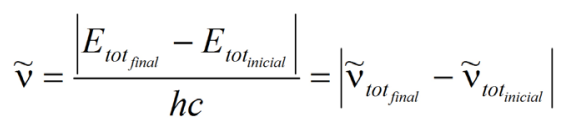

altera-se em relação ao seu valor incidente sobre o material. A detecção e a medida destas variações, para cada número de onda $\widetilde{V}$ da radiação incidente, é efetuada e registrada por instrumentos denominados espectrofotômetros, cujos registros são chamados espectros. Os espectrofotômetros podem ser de absorção ou de emissão (instrumentos distintos) e cada aparelho destina-se a uma determinada faixa de números de onda. As principais faixas de números de onda podem ser vistas na Tabela 2.

\section{Densidades de Energia e Resolução do Aparelho}

Seja $\rho(\widetilde{v}) d \widetilde{v}$ a energia eletromagnética radiante com número de onda entre $\widetilde{v}$ e $\widetilde{v}+d \widetilde{v}$, por unidade de

Tabela 1 - Parâmetros espectroscópicos de algumas moléculas diatômicas no estado fundamental.

\begin{tabular}{|l|l|l|l|l|l|l|l|}
\hline Molécula & $\widetilde{B}_{e} / \mathbf{c m}^{-1}$ & $\widetilde{\alpha}_{e} / \mathbf{c m}^{-1}$ & $\widetilde{D} / \mathbf{c m}^{-1}$ & $\widetilde{\mathrm{v}}_{e} / \mathbf{c m}^{-1}$ & $\widetilde{x}_{e} \widetilde{\mathrm{v}}_{e} / \mathbf{c m}^{-1}$ & $R_{e}(\mathbf{v}=\mathbf{0}) / \mathbf{p m}$ & $D_{0} / \mathbf{k J} \cdot \mathbf{m o l} \mathbf{l}^{-\mathbf{1}}$ \\
\hline $\mathrm{H}_{2}$ & 60,853 & 3,0622 & $4,71 \times 10^{-2}$ & 4401,213 & 121,336 & 74,14 & 432,1 \\
\hline $\mathrm{H}^{19} \mathrm{~F}$ & 20,9557 & 0,798 & $2,15 \times 10^{-3}$ & 4138,320 & 89,880 & 91,68 & 566,2 \\
\hline $\mathrm{H}^{35} \mathrm{Cl}$ & 10,5934 & 0,3072 & $5,32 \times 10^{-4}$ & 2990,496 & 52,819 & 127,46 & 427,8 \\
\hline $\mathrm{H}^{79} \mathrm{Br}$ & 8,4649 & 0,2333 & $3,46 \times 10^{-4}$ & 2648,975 & 45,218 & 141,44 & 362,6 \\
\hline $\mathrm{H}^{128} \mathrm{I}$ & 6,5122 & 0,1689 & $2,07 \times 10^{-4}$ & 2309,014 & 39,644 & 160,92 & 294,7 \\
\hline${ }^{12} \mathrm{C}^{16} \mathrm{O}$ & 1,9313 & 0,0175 & $6,12 \times 10^{-6}$ & 2169,814 & 13,288 & 112,83 & 1070,2 \\
\hline${ }^{14} \mathrm{~N}^{16} \mathrm{O}$ & 1,6719 & 0,0171 & $5,40 \times 10^{-6}$ & 1904,200 & 14,075 & 115,08 & 626,8 \\
\hline${ }^{14} \mathrm{~N}^{14} \mathrm{~N}$ & 1,9982 & 0,0173 & $5,76 \times 10^{-6}$ & 2358,570 & 14,324 & 109,77 & 941,6 \\
\hline${ }^{16} \mathrm{O}^{16} \mathrm{O}$ & 1,4456 & 0,0159 & $4,84 \times 10^{-6}$ & 1580,19 & 11,980 & 120,75 & 493,6 \\
\hline${ }^{19} \mathrm{~F}{ }^{19} \mathrm{~F}$ & 0,8902 & 0,1385 & $3,30 \times 10^{-6}$ & 916,64 & 11,236 & 141,19 & 154,6 \\
\hline${ }^{35} \mathrm{Cl}{ }^{15} \mathrm{Cl}$ & 0,2440 & 0,00149 & $1,86 \times 10^{-7}$ & 559,72 & 2,675 & 198,79 & 239,2 \\
\hline${ }^{79} \mathrm{Br}{ }^{79} \mathrm{Br}$ & 0,0821 & 0,0003187 & $2,09 \times 10^{-8}$ & 325,321 & 1,0774 & 228,11 & 190,1 \\
\hline${ }^{127} \mathrm{I}^{127} \mathrm{I}$ & 0,03737 & 0,0001138 & $4,25 \times 10^{-9}$ & 214,502 & 0,6147 & 266,63 & 148,8 \\
\hline${ }^{35} \mathrm{Cl}{ }^{19} \mathrm{~F}$ & 0,5165 & 0,004358 & $8,77 \times 10^{-7}$ & 786,15 & 6,161 & 162,83 & 252,5 \\
\hline${ }^{23} \mathrm{Na}{ }^{23} \mathrm{Na}$ & 0,1547 & 0,0008736 & $5,81 \times 10^{-7}$ & 159,125 & 0,7255 & 307,89 & 71,1 \\
\hline${ }^{39} \mathrm{~K}^{39} \mathrm{~K}$ & 0,05674 & 0,000165 & $8,63 \times 10^{-8}$ & 92,021 & 0,2829 & 390,51 & 53,5 \\
\hline
\end{tabular}


volume, onde $\rho(\widetilde{v})$ é denominada densidade espectral de energia radiante. Temos, então,

$$
\rho_{t o t}=\int_{0}^{+\infty} \rho(\widetilde{v}) d \widetilde{v} \quad \text { e } \quad I=c \rho_{t o t}
$$

sendo $\rho_{\text {tot }}$ a energia radiante total por unidade de volume, chamada densidade de energia radiante e I é a intensidade de radiação definida no parágrafo anterior. Tanto os espectrofotômetros de absorção como de emissão comparam os valores $\rho_{\text {tot }}=\int_{\tilde{v}+\Delta \tilde{v}}^{\tilde{v}} \rho(\widetilde{v}) d \widetilde{v}$ incidente e emergente, onde $\Delta \widetilde{v}$ diminui quando aumenta a resolução do aparelho.

\section{Os Coeficientes de Einstein}

Para mostrar os importantes conceitos de absorção, emissão espontânea e emissão estimulada de radiação eletromagnética, bem como o de equilíbrio entre estes fenômenos e de interdependência deles no equilíbrio, suponhamos agora uma molécula hipotética à qual correspondam apenas dois níveis de energia total não degenerados, identificados pelos índices 1 e 2, sendo $\mathrm{E}_{2}$ $>\mathrm{E}_{1}$. Numa transição radioativa entre estes dois níveis, $\mathrm{o}$ número de onda da radiação absorvida ou emitida será

$$
\widetilde{v}_{1 \rightarrow 2} \equiv \widetilde{v}_{2 \rightarrow 1} \equiv \widetilde{v}=\frac{E_{2}-E_{1}}{c h}
$$

Sejam $N_{1}$ e $N_{2}$ respectivamente as quantidades de moléculas nos níveis 1 e 2 . Para o processo de absorção (Figura 4) temos então

$$
\underbrace{\frac{d N_{2}(t)}{d t}}_{a b s}=-\underbrace{\frac{d N_{1}(t)}{d t}}_{a b s}=B_{1 \rightarrow 2} N_{1} \rho(\widetilde{v})
$$

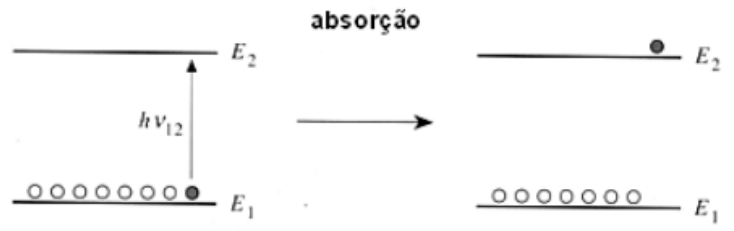

Figura 4 - Uma ilustração do processo de absorção. Um átomo pode absorver luz com energia $h v_{12}=E_{2}-E_{1}$ e ser excitado para um estado de maior energia.

enquanto que para emissão estimulada (Figura 5) temos

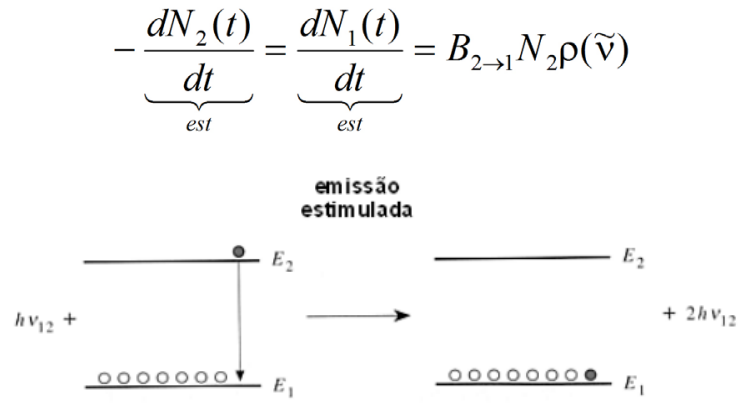

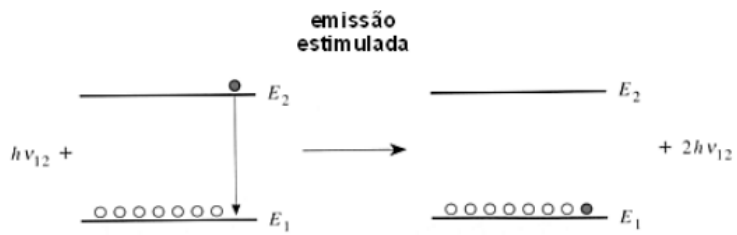

Figura 5 - Uma ilustração do processo de emissão estimulada. Luz com energia $h v_{12}=E_{2}-E_{1}$ pode estimular um átomo em um estado excitado a emitir um fóton $\mathrm{h} v_{12}$ e fazer com que o mesmo retorne ao estado fundamental.

e, para emissão espontânea (Figura 6), temos

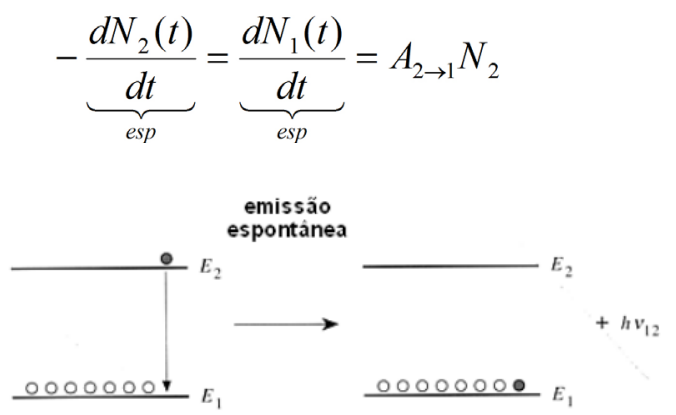

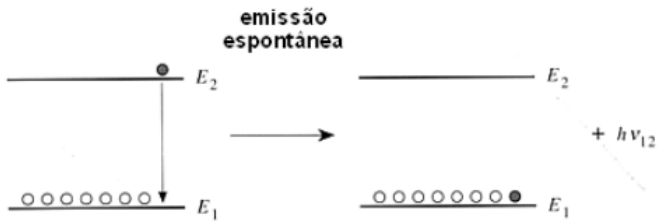

Figura 6 - Uma ilustração do processo de emissão espontânea. Luz com energia $h v_{12}=E_{2}-E_{1}$ pode ser emitida espontaneamente quando um átomo passa de um estado excitado para o estado fundamental.

onde $B_{1 \rightarrow 2}, B_{2 \rightarrow 1}$ e $A_{2 \rightarrow 1}$ são denominados coeficientes de

Tabela 2 - Regiões do espectro eletromagnético e os processos moleculares correspondentes.

\begin{tabular}{|l|l|l|l|l|}
\hline Região & Microondas & $\begin{array}{l}\text { Infravermelho } \\
\text { Distante }\end{array}$ & Infravermelho & $\begin{array}{l}\text { Visível e } \\
\text { Ultravioleta }\end{array}$ \\
\hline Frequência / s & $10^{-1}-10^{11}$ & $10^{11}-10^{13}$ & $10^{13}-10^{14}$ & $10^{14}-10^{16}$ \\
\hline Comprimento de Onda / m & $3 \times 10^{-1}-3 \times 10^{-3}$ & $3 \times 10^{-3}-3 \times 10^{-5}$ & $3 \times 10^{-5}-6,9 \times 10^{-7}$ & $6,9 \times 10^{-7}-2 \times 10^{-7}$ \\
\hline Número de Onda / cm ${ }^{-1}$ & $0,033-3,3$ & $3,3-330$ & $330-14500$ & $14500-50000$ \\
\hline Energia / J. molécula ${ }^{-1}$ & $6,6 \times 10^{-25}-6,6 \times 10^{-23}$ & $6,6 \times 10^{-23}-6,6 \times 10^{-21}$ & $6,6 \times 10^{-21}-2,9 \times 10^{-19}$ & $2,9 \times 10^{-19}-1,0 \times 10^{-18}$ \\
\hline Processo molecular & $\begin{array}{l}\text { Rotação de moléculas } \\
\text { poliatômicas }\end{array}$ & $\begin{array}{l}\text { Rotação de pequenas } \\
\text { moléculas }\end{array}$ & $\begin{array}{l}\text { Vibração de ligações } \\
\text { flexíveis }\end{array}$ & Transições eletrônicas \\
\hline
\end{tabular}


Einstein. Como, no equilíbrio, o número de moléculas em cada nível não pode ser alterado e a razão $N_{2} / N_{1}$ obedece à lei de distribuição de Boltzmann, considerando que o valor de $\rho(\widetilde{v})$ possa ser dado pela equação de Planck para radiação de corpo negro obtém-se

$$
B_{1 \rightarrow 2}=B_{2 \rightarrow 1}=\left(8 h \pi \widetilde{v}^{3}\right)^{-1} A_{2 \rightarrow 1}
$$

Entretanto, como todas as grandezas envolvidas nesta equação manterão seus respectivos valores ao sairmos do equilíbrio, sua validade é geral. Esta equação indica que os coeficientes de Einstein dependem de $\widetilde{V}$.

Lei de Beer-Lambert e Intensidade Integrada de Absorção

\section{Define-se:}

- Adimensional absorbância decimal referente ao número de onda $\widetilde{v}$, dado por $A(\widetilde{v})=\log \left(I_{0}(\widetilde{v}) / I(\widetilde{v})\right.$, onde $I_{0}(\widetilde{v})$ e $I(\widetilde{v})$ são respectivamente as intensidades das radiações incidente e emergente em relação ao material irradiado, ambas referentes ao mesmo número de onda $\widetilde{v}$. Denomina-se transmitância à razão $I(\widetilde{v}) / I_{0}(\widetilde{v})$. Note-se que a absorbância decimal é a grandeza que aparece como ordenada no espectro de absorção obtido durante a experiência a ser efetuada $\mathrm{e}$ que, se neste espectro o valor $\widetilde{V}$ corresponder à ponta de um pico, $\widetilde{v}$ corresponderá ao fundo de um vale no espectro de transmitância da mesma amostra.

- Adimensional absorbância neperiana referente ao número de onda $\widetilde{v}$, dado por $A_{e}(\widetilde{v})=\ln \left(I_{0}(\widetilde{v}) / I(\widetilde{v})\right)$

Lei de Beer-Lambert

$$
I_{0}(\widetilde{v})=I(\widetilde{v}) e^{l c \kappa(\widetilde{v})}=I(\widetilde{v}) 10^{l c \varepsilon(\widetilde{v})}
$$

$c \kappa(\widetilde{v})=N \sigma(\widetilde{v})$, onde:

- l é o comprimento do caminho ótico (distância percorrida pela radiação dentro do material), cuja unidade SI é $\mathrm{m}$.

- $c$ é a concentração molar, cuja unidade SI é mol.m-3 (ou mmol L-1).

- $\kappa(\widetilde{V})$ é o coeficiente neperiano de absorção molar, cuja unidade SI é m2 mol-1.

- $\varepsilon(\widetilde{v})$ é o coeficiente decimal de absorção molar (antigamente denominado coeficiente de extinção), cuja unidade SI é $\mathrm{m}^{2} \mathrm{~mol}^{-1}$.

- $\quad N$ é o número de moléculas absorventes por unidade de volume, cuja unidade SI é $\mathrm{m}^{-3}$.

- $\sigma(\widetilde{V})$ é a seção transversal de absorção, cuja unidade SI é $\mathrm{m}^{2}$.

Intensidade integrada de absorção da n-ésima linha espectral

$$
A_{n}=\int_{0}^{+\infty} \kappa(\widetilde{v}) d \widetilde{v}
$$

cuja unidade SI é m.mol ${ }^{-1}$. Note-se que cada linha é integrada sobre todos os valores possíveis de $\kappa(\widetilde{v})$, independentemente das outras (evidentemente, $\kappa(\widetilde{v}) \neq 0$ somente sobre uma estreita faixa em torno dos números de onda correspondentes aos valores $A(\widetilde{\mathrm{V}})$; não confundir com intensidade de radiação $I$, definida no item 1.3.1).

\section{Eficiência Quântica e Intensidade Integrada de Emissão}

- Adimensional eficiência quântica de fluorescência $\mathrm{q}_{\mathrm{FM}}$, dado pela razão entre as somas, sobre todos os números de onda, dos fótons emitidos e absorvidos pela amostra. Em outras palavras, dado pela razão entre as intensidades emitida e absorvida.

- Espectro molecular de fluorescência $F(\widetilde{V})$, cuja unidade SI é m. Para definí-lo considere, inicialmente, que INT seja o valor daintegral, sobre todos os números de onda, da intensidade relativa da radiação, com número de onda $\widetilde{v}$, emitida pela amostra. $\mathrm{O}$ adjetivo relativa refere-se a qualquer intensidade referencial, escolhida de acordo com a conveniência experimental. Note-se que é uma intensidade relativa a grandeza que aparece, como ordenada, no espectro de fluorescência obtido durante a experiência. Multiplicando-se a intensidade relativa com número de onda $\widetilde{v}$ pela razão $q_{F M} / I N T$, obtém-se $F(\widetilde{v})$.

- A intensidade integrada de emissão da n-ésima linha 
espectral

$$
q_{F M}=\int_{0}^{+\infty} F(\widetilde{v}) d \widetilde{v}
$$

adimensional. Note-se que cada linha éintegrada sobre todos osvalores possíveis de $F(\widetilde{V})$, independentemente das outras (evidentemente, $F(\widetilde{v}) \neq 0$ somente sobre uma estreita faixa de valores em torno dos números de onda correspondentes aos maiores valores $F(\widetilde{v}$ ); não confundir com as intensidades de radiação $I$, definida no item 1.3.1 e integração de absorção $A n$, definida no item 1.3.3).

Regras de Seleção para Espectros Roto-Vibracionais

Regras para Oscilador Harmônico e Rotor Rígido

Algumas transições radioativas são proibidas, por razões explicadas através da mecânica quântica. Por exemplo, dentro do modelo do rotor rígido apenas transições com $\Delta J$ $= \pm 1$, em moléculas com momento de dipolo permanente, absorvem ou emitem radiação. A esta limitação costumase chamar regra de seleção do rotor rígido. Analogamente, a regra de seleção do oscilador harmônico consiste em apenas ocorrerem transições radioativas em moléculas cujo momento de dipolo varie durante a vibração, sendo $\Delta v= \pm 1$. Se os modelos do oscilador harmônico e do rotor rígido, simultaneamente admitidos, representassem alguma molécula diatômica real, seus níveis de energia seria aqueles representados na Figura 1. Para tal molécula, as regras de seleção antes mencionadas seriam rigidamente obedecidas. Fazendo, então, $\widetilde{v}_{e l}$ constante e $\widetilde{x}_{e}=\widetilde{D}=\widetilde{\alpha}_{e}=$ 0 na equação fundamental (item 1.2), para $\Delta J= \pm 1$ e $\Delta v=$ \pm 1 obtemos $\widetilde{v}=\widetilde{v}_{e} \pm 2 \widetilde{B}_{e} J$, sendo $J=1,2,3, \ldots$. Portanto, o espectro roto-vibracional $\left(\widetilde{\mathrm{V}}_{e l}=\right.$ constante $)$ de absorção desta molécula especial apresentaria picos em

$$
\ldots\left(\widetilde{v}_{e}-6 \widetilde{B}_{e}\right),\left(\widetilde{v}_{e}-4 \widetilde{B}_{e}\right),\left(\widetilde{v}_{e}-2 \widetilde{B}_{e}\right),\left(\widetilde{v}_{e}+2 \widetilde{B}_{e}\right),\left(\widetilde{v}_{e}+4 \widetilde{B}_{e}\right),\left(\widetilde{v}_{e}+6 \widetilde{B}_{e}\right), \ldots
$$

ou seja, picos igualmente espaçados em $2 \widetilde{B}_{e} \mathrm{~cm}^{-1}$, salvo os dois centrais, que seriam espaçados em $4 \widetilde{B}_{e} \mathrm{~cm}^{-1}$.

\section{Espectros Reais}

Os espectros roto-vibracionais de absorção de moléculas diatômicas reais em fase gasosa lembram, de fato, aquele descrito no parágrafo "Regras para Oscilador Harmônico e Rotor Rígido", mas dele diferem porque, para que o modelo matemático se aproxime do espectro real, não se pode impor $\widetilde{x}_{e}=\widetilde{D}=\widetilde{\alpha}_{e}=0$, ou seja, deve-se corrigir os modelos do oscilador harmônico e do rotor rígido e considerar a interação vibração-rotação. Quanto mais significativos forem os valores das correções (vê-las na equação fundamental, item 1.2), menos as regras de seleção apresentadas no citado parágrafo serão rigidamente obedecidas. As Figuras 7 e 8 mostram, respectivamente, correções aos níveis energéticos do rotor rígido e do oscilador harmônico. A Tabela 3 mostra a existência, no espectro real do $\mathrm{HCl}$ [14], de pequenos picos devidos aos sobretons, absolutamente proibidos pela regra de seleção do oscilador harmônico.

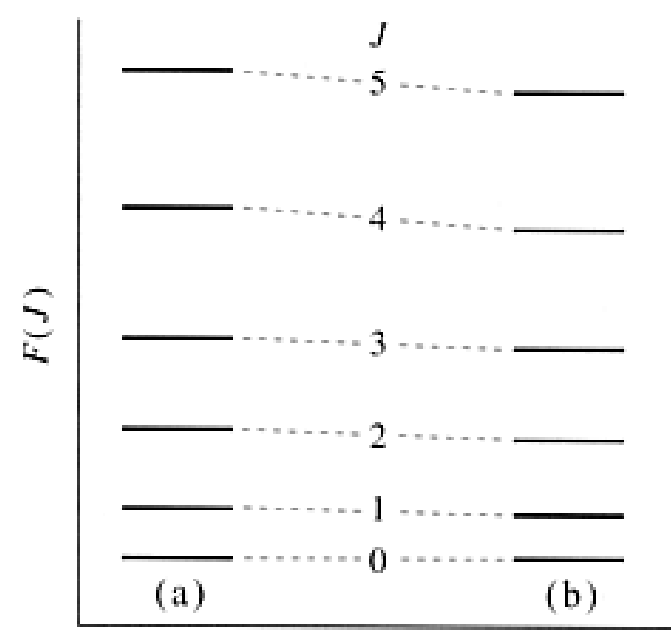

Figura 7 - Níveis rotacionais de um rotor rígido(a) e de um rotor nãorígido (b).

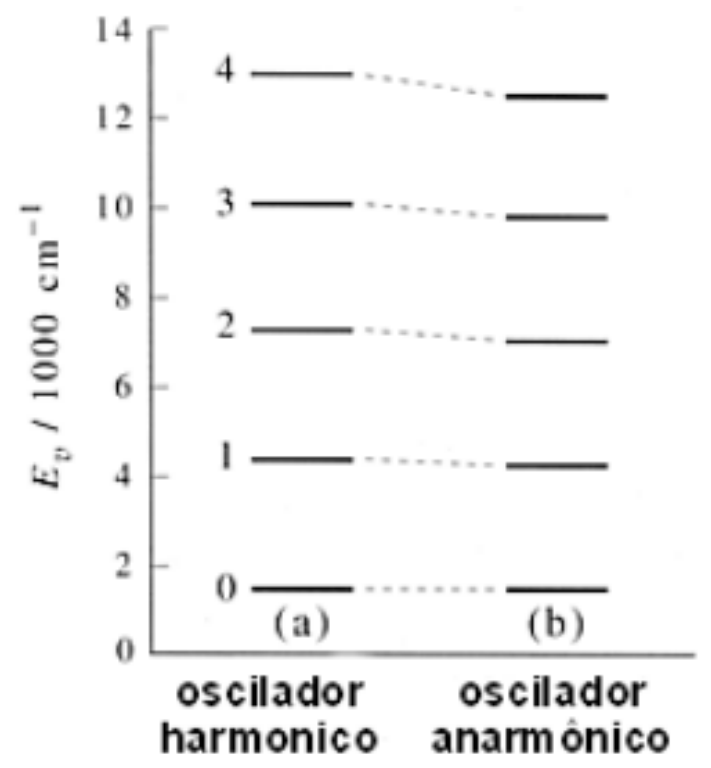

Figura 8 - Estados vibracionais da molécula $\mathrm{H}^{35} \mathrm{Cl}$ calculadas com: a) a aproximação do oscilador harmônico e b) com a correção da anarmonicidade. 
Tabela 3 - O espectro vibracional da molécula $\mathrm{H}^{35} \mathrm{Cl}$.

\begin{tabular}{|c|c|c|c|c|}
\hline & & & $\widetilde{v}_{o b s} / \mathbf{c m}^{-1}$ & \\
\hline \multirow[t]{2}{*}{ Transição } & $\widetilde{v}_{a b s} / \mathbf{c m}^{-1}$ & $\widetilde{V}=2885,9 \mathrm{v}$ & $\widetilde{v}=2885,9 v-52,82 \mathrm{v}(\mathrm{v}+1)$ & $\widetilde{v}_{\text {obs }}(0 \rightarrow \mathrm{v})$ \\
\hline & & & & $\widetilde{v}_{o b s}(0 \rightarrow 1)$ \\
\hline $0 \rightarrow 1$ (fundamental) & 2885,9 & 2885,9 & 2885,3 & 1,000 \\
\hline $0 \rightarrow 2$ (primeiro sobreton) & 5668,0 & 5771,8 & 5665,0 & 1,964 \\
\hline $0 \rightarrow 3$ (segundo sobreton) & 8347,0 & 8657,7 & 8339,0 & 2,892 \\
\hline $0 \rightarrow 4$ (terceiro sobreton) & 10923,1 & 11543,6 & 10907,4 & 3,785 \\
\hline $0 \rightarrow 5$ (quarto sobreton) & 13396,5 & 14429,5 & 13370,2 & 4,642 \\
\hline
\end{tabular}

Espectro Eletrônico: Definições e Aproximações

A Figura 2 mostra diversas curvas de energia potencial eletrônica em função da distância internuclear, cada uma apresentando um diferente valor para $\widetilde{v}_{e l}$ (item 1.2). Para cada curva são mostrados os correspondentes primeiros níveis vibrônicos, não aparecendo os níveis rotovibrônicos porque estes se encontram demasiadamente pouco distanciados entre si para serem representados. Como os valores referentes às energias eletrônicas são muito maiores do que aqueles relativos às energias vibracionais e, estes, muito maiores dos correspondentes às energias rotacionais, ao contrário do que acontece nos espectros roto-vibracionais, nos espectros eletrônicos frequentemente é uma aproximação suficiente ignorar os termos rotacionais da equação fundamental (os últimos dois, item 1.2), assim como ignorados foram os níveis rotovibrônicos na Figura 2 (note-se, entretanto, que precisas atribuições de níveis roto-vibrônicos, nos espectros eletrônicos, são perfeitamente possíveis). A Tabela 4 mostra os valores de $R_{e}$ e de $\widetilde{T}$, correspondentes à Figura 2. A Figura 3 indica que $\widetilde{T}_{e}=\Delta \widetilde{\mathrm{V}}_{e l}$ e mostra o significado de outros parâmetros, inclusive $\mathrm{D}_{0}$ (Tabela 1).

Tabela 4 - Comprimentos de ligação de vários estados eletrônicos da molécula $\mathrm{O}_{2}$.

\begin{tabular}{|c|l|l|}
\hline Estado eletrônico & $\widetilde{T}_{e} / \mathbf{c m}^{-1}$ & $R_{e} / \mathbf{p m}$ \\
\hline${ }^{3} \Sigma_{g}^{-}$ & 0 & 120,74 \\
\hline${ }^{1} \Delta_{g}$ & 7918,1 & 121,55 \\
\hline${ }^{1} \Sigma_{g}^{+}$ & 13195,2 & 122,67 \\
\hline${ }^{3} \Sigma_{u}^{+}$ & 36096,0 & 142,00 \\
\hline${ }^{3} \Sigma_{u}^{-}$ & 49802,0 & 160,00 \\
\hline
\end{tabular}

Potencial de Morse

\section{Definição}

O potencial de Morse

$$
V(l)=D\left(1-e^{-\beta\left(l-l_{0}\right)}\right)^{2},
$$

em que $l_{0}$ é a distância de equilíbrio do oscilador, $l$ é a distância a qualquer momento e $D$ e $\beta$ são parâmetros que dependem da molécula considerada, quando usado na equação de Schrödinger produz os dois termos referentes ao movimento vibracional da equação fundamental, se impusermos que, em unidades de energia,

$$
D=\frac{h c \widetilde{v}_{e}}{4 \widetilde{x}_{e}} .
$$

Neste grau de aproximação, portanto, o potencial de Morse reproduz corretamente o oscilador anarmônico que ocorre na molécula real (note-se que este é o grau de aproximação com que trataremos o espectro eletrônico, conforme vimos no item 1.4.1). Analisando a expressão matemática do potencial de Morse notamos que $V\left(l_{0}\right)=0$ e $V(\infty)=D$. Por isso, a Figura 3 indica que, neste grau de aproximação, $D=D_{e}$.

\section{Constante de Força}

Por outro lado, se fizermos a expansão em série de Maclaurin do potencial de Morse e a truncarmos no termo quadrático, teremos o potencial do oscilador harmônico, proveniente da lei de Hooke $f=-k(l-l)$, onde $k$ é a constante de força da mola (no caso, é a constante de força da ligação). Por isso para o potencial de Morse 
temos, como para o oscilador harmônico,

$$
k=4 \pi^{2} \widetilde{v}_{e}^{2} c^{2} \mu
$$

em unidades de força por comprimento. (Ao utilizar esta equação, é necessário lembrar que, no cálculo da massa reduzida $\mu$ (item 1.2), $m_{1}$ e $m_{2}$ são massas atômicas, não massas molares).

\section{Exatidão em Relação à Experiência}

Quando v aumentar em uma unidade, aos dois termos vibracionais $\mathrm{da}$ equação fundamental (item 1.2) corresponderá a variação de energia

$$
\Delta G=\Delta G(\mathrm{v}+1)-G(\mathrm{v})=h c \widetilde{\mathrm{v}}_{e}\left[1-2 \widetilde{x}_{e}(1+\mathrm{v})\right] .
$$

Como $\widetilde{x}_{e} \ll 1$, para valores pequenos de $\mathrm{v}$ esta variação será positiva. Entretanto, para $\mathrm{v}>\left(2 \widetilde{x}_{e}\right)^{-1}-1$ a variação será negativa, o que é um absurdo. Devemos, então, impor $\mathrm{v}=0,1, \ldots, \mathrm{v}_{\max }$, onde $\mathrm{v}_{\max }$ é o maior inteiro não superior a $\left(2 \widetilde{x}_{e}\right)^{-1}-1$. Este truncamento na série de valores que v pode assumir absolutamente não ocorre no modelo do oscilador harmônico, onde $\mathrm{v} \rightarrow+\infty$, sendo produzido pela correção anarmônica. Substituindo $\mathrm{v}_{\text {fic }}=\left(2 \widetilde{x}_{e}\right)^{-1}-1$ na definição de energia vibracional da molécula $G(v)$ (item 1.2) obtemos

$$
G\left(\mathrm{v}_{f i c}\right)=h c \widetilde{\mathrm{v}}_{e} \frac{1-\widetilde{x}_{e}^{2}}{4 \widetilde{x}_{e}} \approx D_{e}
$$

portanto este valor fictício de v, geralmente não inteiro e superior a $\mathrm{v}_{\max }$, mas quase igual a ele, corresponde a uma energia vibracional quase igual a $D_{\mathrm{e}}$, se o zero de energia vibracional for $E_{e l}$. Isto indica que o truncamento da série de valores de $\mathrm{v}$, em $\mathrm{v}_{\max }$, é coerente com o uso de dois termos vibracionais na equação fundamental e com o uso do potencial de Morse. Sempre que, para uma determinada faixa de valores de v, o gráfico dos valores experimentais de $\Delta \mathrm{G}$ contra $(1+\mathrm{v})$ for, dentro do erro experimental, linear, o potencial de Morse será experimentalmente correto para aquela faixa de valores de v. Tal gráfico é denominado de Birge e Sponer. Entretanto, se o gráfico não for linear, o potencial de Morse e os dois termos vibracionais na equação fundamental não representarão corretamente a energia vibracional da molécula (outros termos, de potência superior à segunda, deveriam ser considerados, o que modificaria a forma do potencial de Morse). Note-se que chamamos de valor experimental de $\Delta \mathrm{G}$ ao módulo da diferença entre dois números de onda de radiações absorvidas ou emitidas, para as quais um dos níveis vibrônicos terminais seja coincidente, enquanto que o outro seja o nível v para uma delas e o nível v+1 para a outra, ambos estes últimos dois pertencendo à mesma curva de energia potencial eletrônica.

\section{Energia de Dissociação}

Como $G\left(\mathrm{v}_{f i}\right) \approx \mathrm{D}_{\mathrm{e}}$, evidentemente $G\left(v_{f i}\right)-G(0) \approx D 0$, ou

$$
D_{0} \approx h c \widetilde{v}_{e} \frac{1-2 \widetilde{x}_{e}}{4 \widetilde{x}_{e}}
$$

Note-se, entretanto, que para se conseguir um valor comparável às energias de dissociação molares obtidas através de outros meios experimentais, a expressão anterior deve ser multiplicada pela constante de Avogadro.

Espectro Eletrônico: Definições e Aproximações

A regra de seleção para transição roto-vibrônica (entre níveis roto-vibrônicos de diferentes curvas de energia potencial eletrônica, porque se a curva fosse a mesma diríamos transição roto-vibracional) é o princípio de Franck-Condon. Desaparecem completamente as restrições, consideradas nos espectros roto-vibracionais, referentes seja ao oscilador harmônico como ao rotor rígido. Portanto, quando os valores de $n$ inicial e final diferirem, mesmo se considerarmos (item 1.3.4) $\widetilde{x}_{e}=\widetilde{D}=\widetilde{\alpha}_{e}=0$ na equação fundamental poderemos ter, para transições radioativas, $\Delta J \neq 1, \Delta v \neq 1$, molécula sem momento de dipolo permanente e molécula cujo momento de dipolo não varie durante a vibração. As Figuras 9, 10 e 11 ilustram o princípio. Algumas características devem ser ressaltadas:

- Salvo para o nível vibrônico fundamental (aquele com $\mathrm{v}=0$ ) de cada curva de energia potencial eletrônica, as separações nucleares mais prováveis ocorrem próximo à compressão e à distensão máximas da ligação (as áreas sombreadas laterais são maiores do que as centrais, nas Figuras 9 e 10).

- Como a velocidade eletrônica é mais do que mil vezes maior do que a nuclear, transições entre diferentes curvas de energia potencial eletrônica podem ser representadas por linhas verticais nas Figuras 9 e 
10, o que é uma consequência trivial da aproximação de Born-Oppenheimer (item 1.1). A intensidade da radiação absorvida ou emitida aumenta com o fator de Franck-Condon, que é o quadrado da integral espacial e de spin do produto das partes vibracionais das funções de onda dos estados vibrônicos terminais considerados (as quais são, aproximadamente, funções de onda de oscilador harmônico). Isto fornece uma rigorosa justificativa matemática ao uso prático das áreas sombreadas das Figuras 9 e $\mathbf{1 0}$.

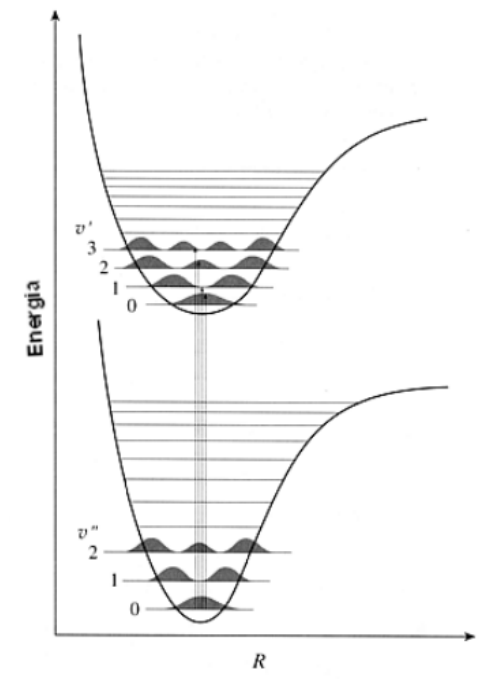

Figura 9 - Duas curvas de potencial mostrando os estados vibracionais associados a cada estado eletrônico. $O$ mínimo da curva superior coincide com o mínimo da curva inferior. As áreas sombreadas correspondem às densidades de probabilidade de cada estado vibracional. As linhas verticais representam transições vibrônicas de $\mathbf{0} \rightarrow \mathbf{v}$ '.

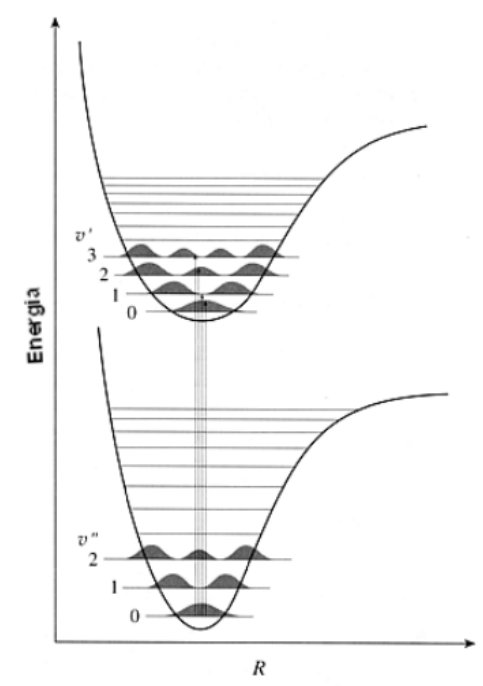

Figura 10 - Duas curvas de potencial mostrando os estados vibracionais associados a cada estado eletrônico. $O$ mínimo da curva superior não coincide com o mínimo da curva inferior. As áreas sombreadas correspondem às densidades de probabilidade de cada estado vibracional. As linhas verticais representam transições vibrônicas de $\mathbf{0} \rightarrow \mathbf{v}$ '.
- Quando duas curvas de energia potencial eletrônica apresentarem valores muito diferentes para $R_{e}$ (ver Tabela 4), a transição entre os dois correspondentes níveis vibrônicos fundamentais $(\mathrm{v}=0)$ poderá ser fraca ou inexistente, ao contrário do que ocorre quando os valores de $\mathrm{R}_{\mathrm{e}}$ forem próximos entre si, ocasião em que tal transição será predominante (p.e., para $\mathrm{I}_{2}$ ela é proibida). A Figura 11 mostra este fato, ao comparar as situações representadas nas Figuras 9 e $\mathbf{1 0}$.

\section{O Espectro Eletrônico de Absorção}

\section{Bandas Quentes}

A substituição das regras de seleção referentes ao oscilador harmônico e ao rotor rígido, pelo princípio de Franck-Condon, indica que a espectroscopia de absorção de luz visível e ultra-violeta fornece informações sobre moléculas diatômicas homonucleares (como, p.e., $\left.I_{2}\right)$ que não apresentam espectro nem de absorção de microonda, nem de luz infravermelha. Geralmente, considera-se que na temperatura ambiente apenas o nível vibrônico fundamental $(\mathrm{v}=0)$ da curva de energia potencial eletrônica fundamental $(n=0)$ encontra-se significativamente povoado. Em alguns casos, entretanto, isto não é verdade. Em tais casos aparecem, então, as chamadas bandas quentes, correspondentes a absorções que partem de níveis que apresentam $n=0$ mas $\mathrm{v} \geq 1$. $\mathrm{O}$ estudo de tais bandas também fornece informações sobre a estrutura molecular, como acontece, p.e., para o $I_{2}$.

\section{Obtenção de Constantes Espectroscópicas para o $\mathrm{I}_{2}$}

\section{Histórico}

A transição entre a curva de energia potencial eletrônica fundamental e a primeira curva excitada do $\mathrm{I}_{2}$, produz um dos sistemas de bandas espectrais mais intensamente estudados pela espectroscopia molecular. Dentro da vasta bibliografia disponível, podemos destacar a obtenção de funções de onda vibracionais exatas [18], em relação aos erros experimentais dos números de onda e das intensidades de sessenta linhas do espectro de fluorescência [17] e, principalmente, a atribuição dos níveis roto-vibrônicos terminais correspondentes a 719 linhas espectrais de absorção, junto com a obtenção de valores seguros para as constantes espectroscópicas referentes à curva excitada 
[15].

\section{Constantes para a Curva de Energia Potencial Eletrônica Excitada}

Por se tratar de espectro tão bem conhecido, já faz quase quatro décadas que experiências com o espectro eletrônico do $I_{2}$ são usadas no ensino a nível de graduação [11]. O tipo de convergência, com o aumento de $\mathrm{v}$, das energias absorvidas na banda vibracional fundamental, referente à curva de energia potencial eletrônica excitada da molécula $\mathrm{I}_{2}$ (Figuras 12 e 13), permite a fácil obtenção de valores razoavelmente exatos para as constantes espectroscópicas referentes a esta curva. Isto porque, a partir do conhecimento do espectro de absorção e da atribuição dos estados vibrônicos terminais correspondentes a linhas próximas ao limite de convergência, o uso da extrapolação linear de Birge e Sponer [5, páginas de 438 a 441] produz resultados bem aceitáveis para o valor D0 correspondente a tal curva $[4,8,13]$.

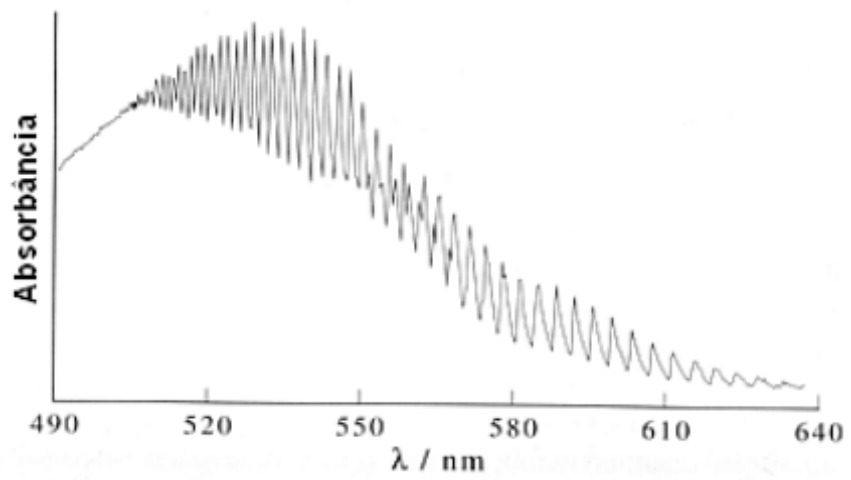

Figura 12 - Espectro de absorção do $I_{2}$ (g) na região do visível.

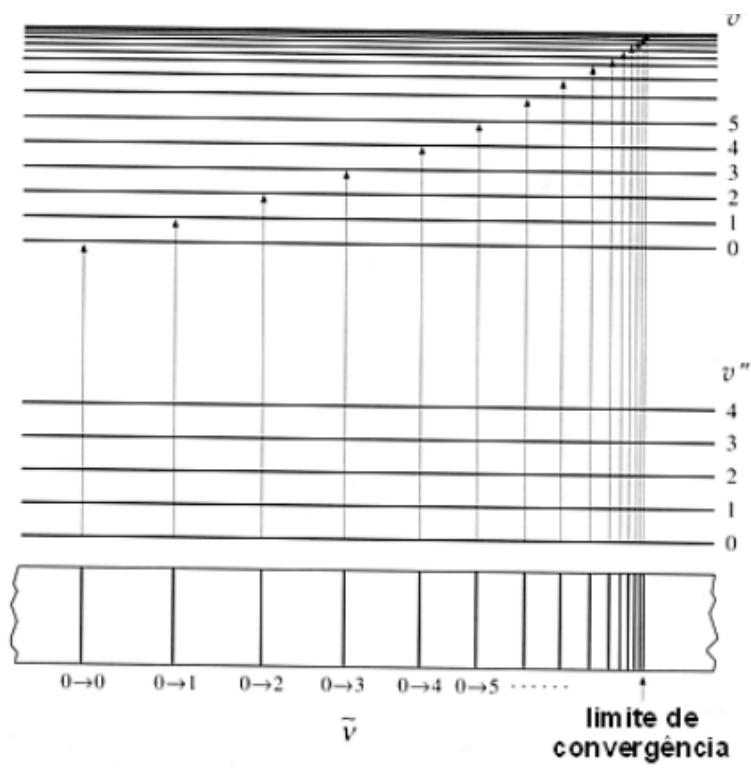

Figura 13 - Espectro eletrônico devido as transições $v^{\prime \prime}=0 \rightarrow v^{\prime}=0,1,2, \ldots$
Em outras palavras, o gráfico de Birge e Sponer (item 1.4.2) é, dentro do erro experimental, linear nesta região, validando o uso do potencial de Morse, permitindo o cálculo de $\widetilde{v}_{e}$ e $\widetilde{x}_{e}$ através da expressão matemática de $\Delta \mathrm{G}$ em função de $(v+1)$ e fornecendo o valor de vfic, através da interseção da reta com o eixo horizontal (onde $\Delta \mathrm{G}=0$; conhecido $\mathrm{v}_{f c}$, teremos $\mathrm{D}_{\mathrm{e}}$ e $\left.\mathrm{D}_{0}\right)$. Se, no índice $\mathrm{x}, \mathrm{y}, \mathrm{x}$ representar $\mathrm{n}$ e $\mathrm{y}$ for $\mathrm{v}$, o método consiste em lembrar que

$\Delta G=G(\mathrm{v}+1)-G(\mathrm{v}) \approx \widetilde{v}_{1,(\mathrm{v}+1) \leftarrow 0, a}-\widetilde{\mathrm{v}}_{1, \mathrm{v} \leftarrow 0, a}=\widetilde{\mathrm{v}}_{1,(\mathrm{v}+1) \leftarrow 1, \mathrm{v}}$

para $\mathrm{v}=0,1, \ldots$. No espectro de absorção da molécula de iodo temos $a=0$ mas, também, duas bandas quentes (item 1.4.4) respectivamente com $\mathrm{a}=1 \mathrm{e} \mathrm{a}=2$.

\section{Constantes para a Curva de Energia Potencial Eletrônica Fundamental}

Para a curva de energia potencial eletrônica fundamental, o método normal (item 1.4.5) de se obter tais constantes seria analisar o espectro de fluorescência resultante de excitação, através da radiação monocromática mais adequada, da molécula de iodo. Manejar um espectro de emissão é, porém, difícil, seja experimental como analiticamente. Isto é particularmente verdadeiro no caso do $\mathrm{I}_{2}$. Aparentemente, portanto, numa experiência a nível de graduação as constantes espectroscópicas mais importantes, que são aquelas referentes à curva fundamental, não seriam obteníveis sem que alguma informação suplementar, extraída da literatura, fosse adicionada àquelas provenientes do espectro de absorção.

Entretanto, já faz doze anos foi mostrado [12] que, utilizando as bandas quentes de um espectro de absorção com suficiente resolução, constantes espectroscópicas razoavelmente precisas podem ser obtidas para a curva fundamental. Se, novamente, no índice $\mathrm{x}, \mathrm{y}, \mathrm{x}$ representar $n$ e y for $\mathrm{v}$, o método consiste em notar que

$$
\widetilde{v}_{1, \mathrm{v} \leftarrow 0,(a+1)}-\widetilde{v}_{1, \mathrm{v} \leftarrow 0, a}=\widetilde{\mathrm{v}}_{0,(a+1) \leftarrow 0, a}, \text { para } \mathrm{v}=0,1, \ldots
$$

A Figura 3 torna a equação anterior ainda mais clara. Deve-se novamente lembrar que, no espectro de absorção da molécula de iodo, temos $a=0$ mas, também, duas bandas quentes (item 1.4.4) respectivamente com $a=1 \mathrm{e}$ $a=2$, conforme indica a Figura 4. 


\section{O Espectro Eletrônico de Absorção}

\section{Relaxação e Supressão Interna}

Os espectros eletrônicos moleculares de emissão são obtidos em aparelhos denominados espectrofluorímetros. Nestes, a radiação emergente é colhida em ângulo reto em relação à radiação incidente, diferentemente do que ocorre nos espectrofotômetros de absorção, onde o ângulo é o dobro disto. A transição da curva de energia potencial eletrônica fundamental para a primeira excitada, chamada excitação, geralmente é radioativa. A transição oposta, chamada relaxação, pode ser radioativa ou não. Quando o material for uma substância pura sob forma de gás muito rarefeito, a relaxação será radioativa e correspondente aos espalhamentos Rayleigh (principalmente) e Raman, ou seja, ocorrerá na faixa de números de onda em que acontece $\mathrm{a}$ absorção, sendo às vezes chamada fluorescência ressonante. Neste caso, aumentando-se a concentração do gás, aumenta-se a intensidade da radiação emitida.

Entretanto, à medida que a concentração volumétrica das partículas continuar a crescer, aumentará o número de colisões entre partículas por unidade de tempo e de volume, e de partículas com as paredes do recipiente por unidade de tempo e de área, ou seja, aumentará a probabilidade da partícula relaxar, pelo menos parcialmente, por transição não radioativa, porque crescerá a possibilidade desta última ocorrer antes da transição radioativa. Por isso, partindose de um gás suficientemente diluído, o aumento de sua concentração inicialmente não altera o número de onda da radiação emitida e aumenta a sua intensidade, mas depois pode modificar o número de onda e até mesmo diminuir a intensidade da radiação emitida (a esta diminuição chama-se supressão).

A Tabela 5 relaciona os diversos tipos de processos de relaxação que uma molécula diatômica excitada em estado gasoso pode sofrer.

Todos os processos não radiativos nela incluídos recebem a denominação genérica de supressão interna. Nesta tabela, $S$ indica singlete (multiplicidade igual a um, logo spin total molecular igual a zero), $T$ representa triplete (multiplicidade igual a três, logo spin total molecular igual a um) e os índices 0 e 1 correspondem respectivamente a curvas de energia potencial eletrônica fundamental e excitadas. Note-se que, nela, apenas a fluorescência e a fosforescência são processos radioativos, sendo a fluorescência bem mais rápida. Finalmente, ressalte-se que os tempos nela listados referem-se a moléculas diatômicas em fase gasosa sob pressão, não valendo, portanto, para gases rarefeitos. A Figura 14 mostra os níveis energéticos inicial e final a que se referem os processos da Tabela 5.
Tabela 5 - Escalas temporais típicas de vários processos pelos quais uma molécula em um estado eletrônico excitado pode relaxar.

\begin{tabular}{|l|l|l|l|}
\hline Processo & Transição & $\begin{array}{l}\text { Mudança } \\
\text { na multi- } \\
\text { plicidade }\end{array}$ & $\begin{array}{l}\text { Escala de } \\
\text { tempo }\end{array}$ \\
\hline Fluorescência & $\begin{array}{l}\text { Radiativa } \\
\mathrm{S}_{1} \rightarrow \mathrm{S}_{0}\end{array}$ & 0 & $10^{-9} \mathrm{~s}$ \\
\hline Conversão Interna & $\begin{array}{l}\text { Colisão } \\
\mathrm{S}_{1} \rightarrow \mathrm{S}_{0}\end{array}$ & 0 & $10^{-7}-10^{-12} \mathrm{~s}$ \\
\hline Relaxação ibracional & $\mathrm{Colisional}$ & & $10^{-14} \mathrm{~s}$ \\
\hline $\begin{array}{l}\text { Cruzamento } \\
\text { Intersistema }\end{array}$ & $\mathrm{S}_{1} \rightarrow \mathrm{T}_{1}$ & 1 & $10^{-12}-10^{-6} \mathrm{~s}$ \\
\hline Fosforescência & $\mathrm{T}_{1} \rightarrow \mathrm{S}_{0}$ & 1 & $10^{-7}-10^{-5} \mathrm{~s}$ \\
\hline $\begin{array}{l}\text { Cruzamento } \\
\text { Intersistema }\end{array}$ & $\mathrm{T}_{1} \rightarrow \mathrm{S}_{0}$ & 1 & $10^{-8}-10^{-3} \mathrm{~s}$ \\
\hline
\end{tabular}

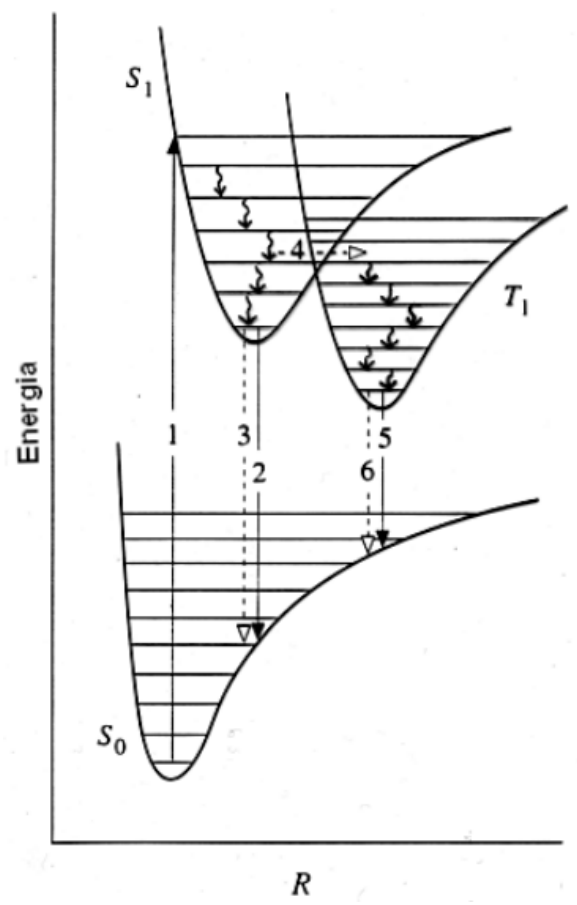

Figura 14 - Esquema de um processo de absorção e posterior decaimento radiativo e não radiativo para uma molécula diatômica: 1 = absorção, 2 = fluorescência, 3 = conversão interna, 4 = cruzamento intersistema, 5 = fosforescência, $\mathbf{6}$ = cruzamento intersistema.

\section{Outras Supressões}

A supressão interna foi explicada no item 1.6.1. Outras supressões podem, entretanto, ser produzidas por:

- Impurezas, mesmo que em quantidades muito pequenas.

- Formação de exímero D1, que é um dímero excitado que ao relaxar se dissocia, formado pela reação $S_{1}$ $+\mathrm{S}_{0} \rightarrow \mathrm{D}_{1}$ (veja esta simbologia no item 1.6.1). $\mathrm{D}_{1}$ se comporta como uma espécie molecular distinta, 
mostrando propriedades fotofísicas próprias, como por exemplo sua fluorescência específica.

- Auto-absorção, quando o espectro de absorção e o de fluorescência apresentarem superposição, mesmo que parcial.

Por exemplo, para hidrocarbonetos aromáticos policíclicos em solução líquida muito diluída ocorre total relaxação vibracional, mas a supressão da fluorescência que se origina de emissão do estado vibrônico fundamental da curva de energia potencial eletrônica excitada, só acontece a partir de determinada concentração, por causa de formação de exímero e de auto-absorção (antes de se atingir esta concentração, a fluorescência aumenta com a concentração). Note-se que, no estado líquido, a variação de concentração de soluto praticamente não altera o número de choques que cada molécula excitada de soluto sofre, logo não altera a relaxação vibracional, mas modifica a composição da camada de moléculas que envolve cada molécula excitada de soluto, o que afeta a formação de exímero e a auto-absorção. Como outro exemplo, quantidades mínimas da impureza oxigênio suprimem praticamente por completo o espectro de emissão do iodo gasoso. Estes fatos mostram, portanto, como pode ser complicada a interpretação das intensidades de fluorescência e de fosforescência dos diversos sistemas, pois para se determinar que tipo de aproximação pode ou não ser feita, cada caso específico deve ser individualmente estudado.

\section{Espectro de Fluorescência e de Excitação}

O instrumento a ser utilizado é um espectrofluorímetro que contém uma fonte de excitação que emite de modo contínuo, por isso denominado foto-estacionário. Haverá, então, uma população constante de espécies no estado eletrônico excitado que, ao cair para o estado fundamental, poderá emitir radiação. O estado excitado deverá, para que a emissão seja uma fluorescência, apresentar a mesma multiplicidade do estado fundamental. Dois tipos totalmente distintos de espectros podem ser obtidos através deste instrumento:

\section{Espectro de Fluorescência}

Para se obter o espectro de fluorescência, em geral costumase escolher como número de onda para a excitação aquele em que a amostra absorver com maior intensidade no seu espectro de absorção, mantendo-se fixo este número ao se registrar os números de onda e as intensidades das emissões.

\section{Espectro de Excitação}

Por outro lado, pode-se apenas registrar emissões que ocorram num número de onda fixo, geralmente aquele em que a amostra emitir com maior intensidade, enquanto varia-se o número de onda de excitação sobre toda a faixa do seu espectro de absorção. Embora em ambos os casos o registro seja o de emissões por fluorescência, convencionase chamar espectro de fluorescência apenas ao primeiro, denominando-se espectro de excitação ao segundo. Este deve, portanto, apresentar picos nos mesmos números de onda do espectro de absorção e, além disto, Ter forma semelhante a este último. Note-se que, ao contrário do que geralmente ocorre, no espectro de excitação a intensidade e o número de onda não se referem à mesma radiação (a primeira refere-se à radiação emitida pela amostra, enquanto que o segundo à absorvida).

\section{Posições Relativas das Linhas}

Considerando válidos para os meios condensados os tempos de relaxação vibracional e de fluorescência vistos na Tabela 5, a fluorescência ocorrerá sempre a partir do nível vibrônico fundamental de uma curva de energia potencial eletrônica excitada. Supondo inexistência de bandas quentes, a absorção ocorrerá sempre a partir do nível vibrônico fundamental da curva de energia potencial eletrônica fundamental. Por isso, quando ambas estas duas afirmações forem válidas, o espaçamento entre os picos dos espectros de absorção e de fluorescência refletirão, respectivamente, o espaçamento entre os níveis vibrônicos das curvas de energia potencial eletrônica excitada e fundamental e, além disto, o espectro de fluorescência se encontrará em números de onda menores do que o de absorção (a não ser por no máximo uma linha coincidente, como veremos no item 1.6.5).

\section{Linha Coincidente e Imagem Especular}

Seja $R_{e}(n)$ a distância internuclear correspondente ao mínimo da curva de energia potencial eletrônica identificada por ne represente-se por $(n, \mathrm{v})$ ao nível vibrônico $\mathrm{v}$ da curva $\mathrm{n}$. Considere-se que as duas afirmações feitas no item 1.6.4 sejam válidas e que, além disto, $\operatorname{Re}(0) \approx$ $\operatorname{Re}\left(n^{\prime}\right)$, sendo $n^{\prime} \neq 0$. A adição desta última restrição obriga 
a que o espectro de absorção apresente uma e somente uma linha cuja faixa de números de onda coincida, pelo menos parcialmente, com a faixa de números de onda de uma linha do espectro de fluorescência. Evidentemente, tal linha coincidente refere-se à transição radioativa entre os níveis vibrônicos fundamentais $(0,0)$ e $(n, 0)$. Portanto se, em acréscimo a tudo isto, ainda o valor de $\widetilde{v}_{e}$ for aproximadamente o mesmo para as duas curvas $n=0$ e $n$ = n', cada espectro parecerá quase a imagem especular do outro no que se refere aos números de onda dos picos, como na Figura 15 (não comentaremos as intensidades dos picos). Isto costuma ser válido para hidrocarbonetos aromáticos policíclicos em solução líquida diluída.

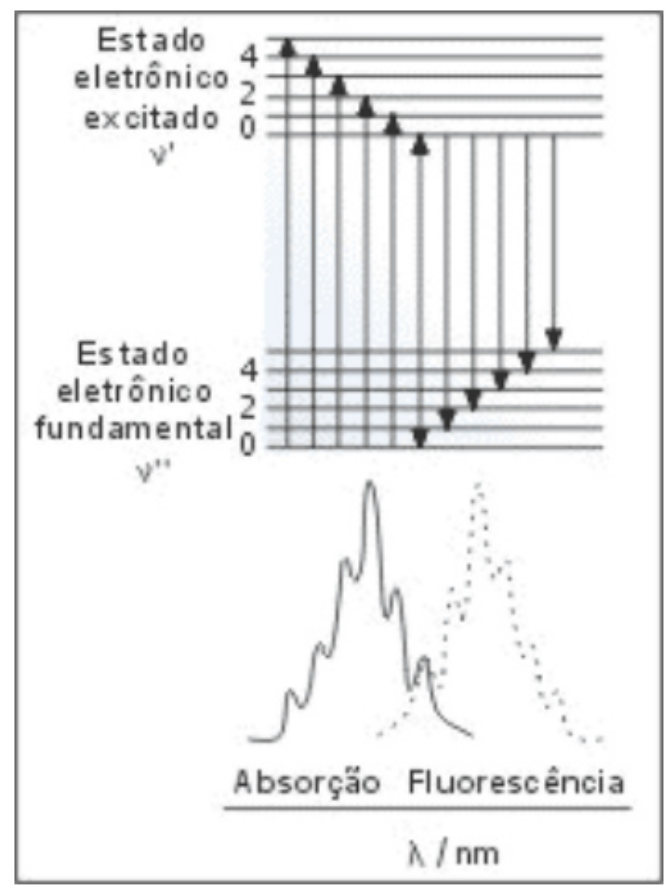

Figura 15 - A imagem especular dos espectros de absorção e de fluorescência e as respectivas transições.

Referências Bibliográficas

1. Stafford, F. E.; Holt, C. W.; Paulson, G. L., Vibration-Rotation Spectrum of $\mathrm{HCl}, \mathrm{J}$. Chem. Educ., 1963, 40: 245-249.

2. Zare, R. N., Calculation of Intensity Distribution in the Vibrational Structure of Electronic Transitions: The Ressonance Series of Molecular Iodine, J. Chem. Phys., 1964, 40: 1934-1944.

3. Verma, R. D.; Ultraviolet Ressonance Spectrum of the Iodine Molecule, J. Chem. Phys., 1960, 32: 738-
749.

4. Steinfeld, J. I.; Zare, R. N.; Jones, L.; Lesk, M.; Klemperer, W., Spectroscopic Constants and Vibrational Assignment for the State of Iodine, J. Chem. Phys., 1965, 42: 25-33. (A lista das 719 linhas medidas e relacionadas aos correspondentes vibrônicos não se encontra no artigo, tendo sido depositada como Documento 8187 na Biblioteca do Congresso dos EUA. Cópias podem ser obtidas mediante pagamento de taxa).

5. Schoemaker, D. P.; Garland, C. W.; Nibler, J. W., Experiments in Physical Chemistry, McGraw-Hill Book, New York, 5a. edição, 1989.

6. Herzberg, G., Molecular Spectra and Molecular Structure, Vol. 1. Spectra of Diatomic Molecules, D. Van Nostrand, New York, 2a. edição, 1950. (Junto com seus outros volumes este livro, cuja primeira edição data de 1939 é, com justiça, o tratado básico da espectroscopia).

7. D'alterio, R.; Mattson, R.; Harris, R., Potential Curves for the I2 Molecule, J. Chem. Educ., 1974, 51: 282-284.

8. McNaught, I. J., The Electronic Spectrum of Iodine Revisited, J. Chem. Educ., 1974, 57: 101-105.

(A equação (8) desse artigo contém um erro. Deve-se substituir $(\mathrm{v}+1)$ por $\mathrm{v})$.

9. Stafford, F. E., Band Spectra and Dissociation Energies, J. Chem. Educ., 1962, 39: 626-629.

10. Snadden, R. B., The Iodine Spectrum Revisited, J. Chem. Educ., 1987, 64: 919-921. 